\title{
Sur le troisième invariant d'une forme quadratique
}

\section{J. BARGE}

École Polytechnique, F-91128 Palaiseau

M. OJANGUREN

Section de Mathématiques, UNIL, CH-1015 Lausanne

Résumé: Nous étudions un invariant à valeurs dans un sous-quotient de $K_{3}$.

Mots clef: invariant, Arason, Clifford, Giffen, Ketu, groupe de Steinberg, formes quadratiques, cohomologie des groupes.

\section{Introduction}

Dans [1] Giffen montre comment associer à toute matrice $\alpha$ symétrique élémentaire à coefficients dans un anneau commutatif $A$, un élément $s_{2}(\alpha)$ dans un quotient convenable de $K_{2}$. Cet invariant $s_{2}(\alpha)$ est très concret: c'est l'obstruction à relever $\alpha$ en un élément symétrique du groupe de Steinberg de $A$. Il est démontré dans [10] qu'en composant $s_{2}$ avec l'homomorphisme de Hurewicz et la classe de Chern $c_{22}$ définie par Grothendieck, on obtient l'invariant de Clifford $e_{2}(\alpha) \in \mathrm{H}^{2}\left(A, \mu_{2}\right)$.

Dans [3], par des méthodes homotopiques, Giffen a construit, pour tout entier naturel $n$, un invariant $s_{n}(\alpha)$, défini sur le noyau des précédents, à valeurs dans un quotient de $K_{n}(A)$.

Dans cet article nous définissons $s_{3}$ de façon purement algébrique et étudions, dans le cas où $A$ est un corps, ses liens avec l'invariant d'Arason.

\section{Un invariant cohomologique}

Soit $G$ un groupe muni d'une action d'un groupe $\Gamma$. Supposons que les groupes d'homologie entière $H_{i}(G)$ soient nuls pour tout $i$ positif inférieur à $n$. Le groupe de cohomologie $H^{n}\left(G, H_{n}(G)\right)$ est alors (d'après [4], par exemple) canoniquement isomorphe à $\operatorname{Hom}\left(H_{n}(G), H_{n}(G)\right)$. Notons $u$ la classe de cohomologie correspondante à l'identité. Elle est, bien sûr, invariante par tout automorphisme de $G$. Soit $G \rtimes \Gamma$ le produit semidirect de $\Gamma$ par $G$ et $s_{0}: \Gamma \rightarrow G \rtimes \Gamma$ sa section canonique. On déduit immédiatement du théorème 2 de $[5]$ la suite exacte

$$
0 \longrightarrow H^{n}\left(\Gamma, H_{n}(G)\right) \stackrel{s_{0}^{*}}{\leftrightarrows} H^{n}\left(G \rtimes \Gamma, H_{n}(G)\right) \longrightarrow H^{n}\left(G, H_{n}(G)\right)^{\Gamma} \longrightarrow 0
$$

qui montre l'existence d'un unique relèvement $\hat{u} \in H^{n}\left(G \rtimes \Gamma, H_{n}(G)\right)$ de $u$ satisfaisant $s_{0}^{*}(\hat{u})=0$.

Rappelons que l'ensemble de cohomologie non abélienne $H^{1}(\Gamma, G)$ s'identifie à l'ensemble pointé par $s_{0}$ des sections $s: \Gamma \rightarrow G \rtimes \Gamma$ à conjugaison près. Pour tout $x \in H^{1}(\Gamma, G)$ nous définissons

$$
s_{n}(x) \in H^{n}\left(\Gamma, H_{n}(G)\right)
$$

par $s_{n}(x)=s^{*}(\hat{u})$, où $s$ est une section qui représente $x$. Cet élément ne dépend pas du choix de $s$ dans la classe $x$. 
2.1. Le cas $n=1$.

Dans ce cas $G$ est un groupe quelconque et l'application

$$
s_{1}: H^{1}(\Gamma, G) \longrightarrow H^{1}\left(\Gamma, H_{1}(G)\right)
$$

est induite par l'abélianisation.

\subsection{Le cas $n=2$.}

Dans ce cas $G$ est un groupe parfait. La classe $u \in H^{2}\left(G, H_{2}(G)\right)$ est représentée par l'extension universelle

$$
0 \longrightarrow H_{2}(G) \longrightarrow \widetilde{G} \longrightarrow G \longrightarrow 1
$$

L'action de $\Gamma$ sur $G$ se relève en une unique action sur cette extension. Nous avons donc la suite exacte de cohomologie

$$
\cdots \longrightarrow H^{1}(\Gamma, \widetilde{G}) \longrightarrow H^{1}(\Gamma, G) \stackrel{\partial}{\longrightarrow} H^{2}\left(\Gamma, H_{2}(G)\right) \text {. }
$$

Proposition 2.2.1. L'application $s_{2}$ coïncide avec le connectant $\partial$.

Preuve. Nous commençons par déterminer l'application $s_{2}$. Dans le diagramme

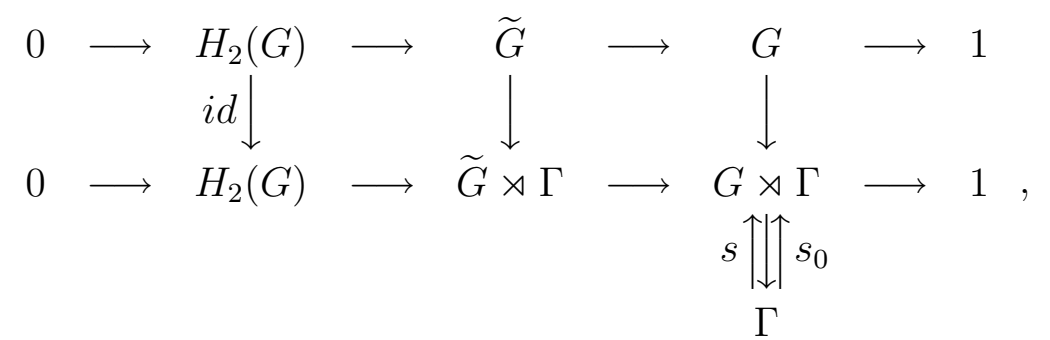

la première suite horizontale représente la classe $u \in H^{2}\left(G \rtimes \Gamma, H_{2}(G)\right)$ et la deuxième la classe $\hat{u}$. Soit

$$
c: G \times G \longrightarrow H_{2}(G)
$$

un 2-cocycle représentant $u$. Ce 2-cocycle n'est pas, contrairement à $u, \Gamma$-invariant. Cela signifie que pour tout $\gamma \in \Gamma$, il existe une application $f_{\gamma}: G \longrightarrow H_{2}(G)$, unique car $\mathrm{G}$ est parfait, telle que pour tout $g_{1}, g_{2} \in G$ on ait l'égalité

$$
\gamma \cdot c\left(\gamma^{-1} \cdot g_{1}, \gamma^{-1} \cdot g_{2}\right)-c\left(g_{1}, g_{2}\right)=f_{\gamma}\left(g_{1} g_{2}\right)-f_{\gamma}\left(g_{1}\right)-f_{\gamma}\left(g_{2}\right) \text {. }
$$

Si $\widetilde{G}$ est ensemblistement identifié à $H_{2} \times G$, l'action de $\Gamma$ sur $\widetilde{G}$ est donnée par la formule

$$
\gamma \cdot(a, g)=\left(a+\gamma \cdot f_{\gamma^{-1}}(g), \gamma \cdot g\right)
$$

pour tout $\gamma \in \Gamma, a \in H_{2}(G)$ et $g \in G$ et la multiplication dans $\widetilde{G} \rtimes \Gamma$, identifié à $H_{2} \times G \times \Gamma$, par la formule

$$
(a, g, \gamma)\left(a^{\prime}, g^{\prime}, \gamma^{\prime}\right)=\left(a+\gamma \cdot a^{\prime}+\gamma \cdot f_{\gamma^{-1}}\left(g^{\prime}\right)+c\left(g, \gamma \cdot g^{\prime}\right), g\left(\gamma \cdot g^{\prime}\right), \gamma \gamma^{\prime}\right)
$$

Soit maintenant $\varphi: \Gamma \longrightarrow G$ un homomorphisme croisé et $s_{\varphi}: \Gamma \longrightarrow G \rtimes \Gamma$ la section qui lui correspond. Il s'agit de calculer la composante en $H_{2}(G)$ de

$$
\left(0, \varphi\left(\gamma_{1} \gamma_{2}\right), \gamma_{1} \gamma_{2}\right)\left(0, \varphi\left(\gamma_{2}\right), \gamma_{2}\right)^{-1}\left(0, \varphi\left(\gamma_{1}\right), \gamma_{1}\right)^{-1}
$$


En utilisant la relation $f_{\gamma_{1} \gamma_{2}}(g)=f_{\gamma_{1}}(g)+\gamma_{1} \cdot f_{\gamma_{2}}\left(\gamma_{1}^{-1} \cdot g\right)$, on trouve

$$
-c\left(\varphi\left(\gamma_{1}\right), \gamma_{1} \cdot \varphi\left(\gamma_{2}\right)\right)-\gamma_{1} \cdot f_{\gamma_{1}^{-1}}\left(\varphi\left(\gamma_{2}\right)\right) \text {, }
$$

qui est l'expression d'un 2-cocycle $\Gamma \times \Gamma \longrightarrow H_{2}(G)$ dont la classe est $s_{2}([\varphi]),[\varphi] \in$ $H^{1}(\Gamma, G)$ étant la classe de $\varphi$.

Pour déterminer $\partial[\varphi]$ on calcule, dans $\widetilde{G}$,

$$
\left(0, \varphi\left(\gamma_{1} \gamma_{2}\right)\right)\left(\gamma_{1} \cdot\left(0, \varphi\left(\gamma_{2}\right)\right)^{-1}\right)\left(0, \varphi\left(\gamma_{1}\right)\right)^{-1}
$$

et on constate que sa première composante coïncide avec le cocycle qui représente $s_{2}([\varphi])$.

\section{Applications aux formes quadratiques}

Soit $A$ un anneau commutatif avec unité dans lequel 2 est inversible. Soit $G L_{n}(A)$ le groupe des matrices $n \times n$ inversibles à coefficients dans $A, E_{n}(A)$ le sous-groupe distingué de $G L_{n}$ engendré par les matrices élémentaires, $G L(A)$ et $E(A)$ les limites inductives des $G L_{n}(A)$ et des $E_{n}(A)$. La donnée d'une forme quadratique $q$ non dégénérée sur $A^{n}$ permet de définir une involution $\alpha \mapsto \alpha^{*}$ sur $G L_{n}(A)$ qui envoie toute matrice sur l'inverse de son adjointe par rapport à $q$. La donnée d'une suite de matrices symétriques $h_{n}$ compatibles avec les inclusions $G L_{n}(A) \hookrightarrow G L_{n+k}(A)$ permet donc de définir une action de $\mathbb{Z} / 2 \mathbb{Z}$ sur $G L(A)$ qui respecte $E(A)$. Avec ces notations, le groupe $H^{0}(\mathbb{Z} / 2 \mathbb{Z}, G L(A))$ s'identifie au groupe $O(A)$, limite inductive des groupes orthogonaux $O_{n}\left(A, h_{n}\right)$. L'ensemble $H^{1}\left(\mathbb{Z} / 2 \mathbb{Z}, G L_{n}(A)\right)$ s'identifie, par la multiplication à gauche par $h_{n}$, à l'ensemble des classes d'isométrie d'espaces quadratiques libres de rang $n$ sur $A$. Si $q$ est un tel espace, on notera $h(q)$ l'élément de $H^{1}(\mathbb{Z} / 2 \mathbb{Z}, G L(A))$ qui lui correspond.

Dans la suite, les formes $h_{m}$ seront les restrictions à $A^{m}$ des formes hyperboliques diagonales de rang $2^{n}$ définies par

$$
h_{2}=\operatorname{diag}(1,-1) \quad \text { et } \quad h_{2^{n}}=\operatorname{diag}\left(h_{2^{n-1}},-h_{2^{n-1}}\right) .
$$

Ainsi, par exemple,

$$
h_{8}=\operatorname{diag}(1,-1,-1,1,-1,1,1,-1) .
$$

\subsection{Le discriminant.}

Soit $K_{1}(A)=G L(A) / E(A)=G L(A) /[G L(A), G L(A)]$. L'application

$$
s_{1}: H^{1}(\mathbb{Z} / 2 \mathbb{Z}, G L(A)) \longrightarrow H^{1}\left(\mathbb{Z} / 2 \mathbb{Z}, H_{1}(G L(A))\right)=H^{1}\left(\mathbb{Z} / 2 \mathbb{Z}, K_{1}(A)\right)
$$

s'interprète comme un raffinement du discriminant signé. En effet, si $A$ est un corps $k$,

$$
H^{1}\left(\mathbb{Z} / 2 \mathbb{Z}, K_{1}(k)\right)=k^{*} / k^{* 2}
$$

et $s_{1}$ est bien l'application qui associe à tout espace quadratique son discriminant. Le premier invariant $e_{1}$ d'un espace quadratique $q$ se définit par $e_{1}(q)=s_{1}(h(q))$.

\subsection{L'invariant de Clifford.}

Soit

$$
0 \longrightarrow K_{2}(A) \longrightarrow S t(A) \longrightarrow E(A) \longrightarrow 0
$$


l'extension centrale universelle du groupe parfait $E(A)$. D'après la proposition 2.2.1, l'application

$$
s_{2}: H^{1}(\mathbb{Z} / 2 \mathbb{Z}, E(A)) \longrightarrow H^{2}\left(\mathbb{Z} / 2 \mathbb{Z}, H_{2}(E(A))\right)=H^{2}\left(\mathbb{Z} / 2 \mathbb{Z}, K_{2}(A)\right)
$$

coïncide avec le connectant associé à la suite exacte $(*)$. On reconnait donc l'invariant de Giffen [2], étudié dans [10].

Pour tout espace quadratique $q$ tel que $e_{1}(q)=0$, on définit le deuxième invariant $e_{2}(q)$ par $e_{2}(q)=s_{2}(h(q))$.

En utilisant une description explicite du groupe de Steinberg, Giffen a démontré que si $A$ est un corps $k$, cet invariant associe à la forme de Pfister $\ll a, b \gg$ le symbole $\{a, b\} \in K_{2}(k) / 2 K_{2}(k)$. Nous donnons une autre démonstration de ce fait, qui, n'utilisant pas la description par générateurs et relations de $S t(k)$, se généralisera à $s_{3}$.

Proposition 3.2.1. Soit $k$ un corps et

$$
\ll x, y \gg=\operatorname{diag}(1,-x,-y, x y)
$$

une forme de Pfister de rang 4. On a

$$
s_{2}(h(\ll x, y \gg))=\{x, y\} \in K_{2}(k) / 2 K_{2}(k) .
$$

Lemme 3.2.2. Notons $\widetilde{\mathbb{Z}}$ le $\mathbb{Z} / 2 \mathbb{Z}$-module $\mathbb{Z}$ muni de l'action donnée par le changement de signe et $G_{n}$ le produit semidirect de $\mathbb{Z} / 2 \mathbb{Z}$ par $\widetilde{\mathbb{Z}}^{n}$, de sorte que l'on a la suite exacte scindée

$$
0 \longrightarrow \widetilde{\mathbb{Z}}^{n} \stackrel{i}{\longrightarrow} G_{n} \longrightarrow \mathbb{Z} / 2 \mathbb{Z} \longrightarrow 0 \text {. }
$$

Soient $A$ un $\mathbb{Z} / 2 \mathbb{Z}$-module quelconque et $u \in H^{p}\left(G_{n}, A\right)$ une classe de cohomologie telle que $i^{*}(u)=0 \in H^{p}\left(\widetilde{\mathbb{Z}}^{n}, A\right)$. Pour tout $n \geq 1$ et tout $p \geq 1$ les conditions suivantes sont équivalentes:

i) $u=0$

ii) $s^{*}(u)=0$ pour toute section $s: \mathbb{Z} / 2 \mathbb{Z} \longrightarrow G_{n}$ sauf pour celle donnée par $s(1)=$ $(1, \ldots, 1)$ et ses conjuguées.

Preuve. Ce lemme est vide pour $n=0$ et la démonstration s'obtient par une double récurrence sur $n$ et sur $p$. Si $p=1$, l'assertion résulte immédiatement de la suite exacte scindée

$$
0 \longrightarrow H^{1}(\mathbb{Z} / 2 \mathbb{Z}, A) \stackrel{s^{*}}{\leftrightarrows} H^{1}\left(G_{n}, A\right) \longrightarrow H^{1}\left(\mathbb{Z} / 2 \mathbb{Z}^{n}, A\right) \longrightarrow 0 .
$$

Supposons donc $p \geq 2$ et $n \geq 1$.

Considérons le diagramme à lignes exactes scindées

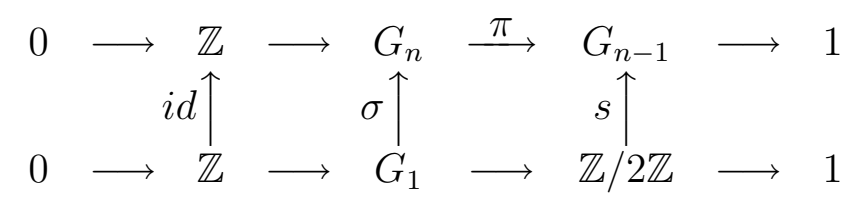

où $\pi$ est la projection qui annule la dernière coordonnée de $\mathbb{Z}^{n}, s: \mathbb{Z} / 2 \mathbb{Z} \longrightarrow G_{n-1}$ est une section quelconque et $\sigma$ un homomorhisme qui rend le diagramme commutatif. Ce diagramme induit en cohomologie un diagramme

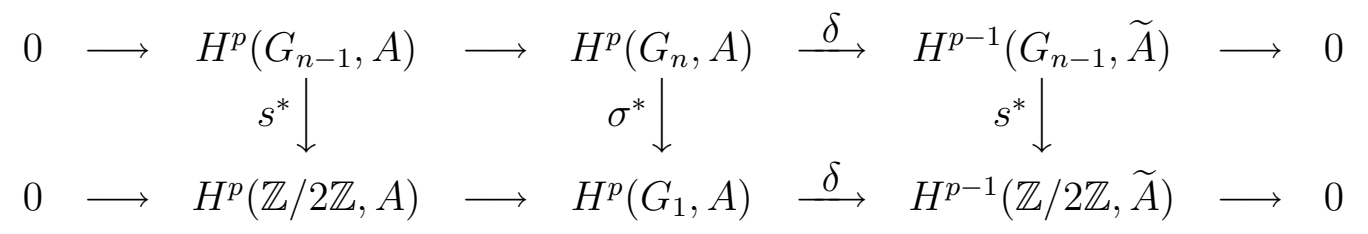


où $\widetilde{A}$ est le $\mathbb{Z} / 2 \mathbb{Z}$-module $H^{1}(\widetilde{\mathbb{Z}}, A)=\operatorname{Hom}_{\mathbb{Z}}(\widetilde{\mathbb{Z}}, A)$ et $\delta$ est l'homomorphisme donné par la suite spectrale de Hochschild-Serre. On déduit par récurrence de ce diagramme qu'il suffit de démontrer le fait suivant: si $p \geq 2$ et $u \in H^{p}\left(G_{1}, A\right)$ est tel que $s^{*}(u)=0$ pour toute section, alors $u=0$.

Le cas $p=2$ se traduit comme ceci: soit

$$
0 \longrightarrow A \longrightarrow \Gamma \stackrel{\pi}{\longrightarrow} G_{1} \longrightarrow 1
$$

une extension de groupes et supposons que toute section $s: \mathbb{Z} / 2 \mathbb{Z} \longrightarrow G_{1}$ se factorise par $\Gamma$; alors l'extension est triviale. Ceci est vrai car $G_{1}=\mathbb{Z} \rtimes \mathbb{Z} / 2 \mathbb{Z}$ est le groupe engendré par $a=(0,1)$ et $b=(1,1)$ avec les seules relations $a^{2}=b^{2}=1$. Les sections définies par $a$ et $b$ se factorisent par $\Gamma$ si et seulement si $\Gamma$ contient deux éléments $\alpha$ et $\beta$ d'ordre 2 tels que $\pi(\alpha)=a$ et $\pi(\beta)=b$ et en ce cas l'extension est scindée par l'homomorphisme $\sigma: G_{1} \longrightarrow \Gamma$ défini par $\sigma(a)=\alpha, \sigma(b)=\beta$.

Soit maintenant $p \geq 3$. En tensorisant sur $\mathbb{Z}$ la suite

$$
0 \longrightarrow \mathbb{Z} \longrightarrow \mathbb{Z}[\mathbb{Z} / 2 \mathbb{Z}] \longrightarrow \widetilde{\mathbb{Z}} \longrightarrow 0
$$

par $A$ on obtient une suite exacte

$$
0 \longrightarrow A \longrightarrow A[\mathbb{Z} / 2 \mathbb{Z}] \longrightarrow \widetilde{A} \longrightarrow 0
$$

qui induit une suite exacte de cohomologie

$$
\cdots \rightarrow H^{p-1}\left(G_{1}, A[\mathbb{Z} / 2 \mathbb{Z}]\right) \rightarrow H^{p-1}\left(G_{1}, \widetilde{A}\right) \rightarrow H^{p}\left(G_{1}, A\right) \rightarrow H^{p}\left(G_{1}, A[\mathbb{Z} / 2 \mathbb{Z}]\right) \rightarrow \cdots
$$

Le lemme de Shapiro ([1], Proposition 4.1.3) montre que $H^{i}\left(G_{1}, A[\mathbb{Z} / 2 \mathbb{Z}]\right)$, étant isomorphe à $H^{i}(\mathbb{Z}, A)$, est nul dès que $i \geq 2$. On a donc un isomorphisme $H^{p-1}\left(G_{1}, \widetilde{A}\right) \longrightarrow$ $H^{p}\left(G_{1}, A\right)$ qui permet de conclure par récurrence sur $p$.

Lemme 3.2.3. Soit $v \in H^{n}\left(\mathbb{Z}^{n}, H_{n}\left(\mathbb{Z}^{n}\right)\right)$ la "classe tautologique". Pour tout multi-indice $I=\left(i_{1}, \ldots, i_{n}\right)$ où les $i_{\lambda}$ prennent les valeurs 0 ou 1 , notons $s_{I}: \mathbb{Z} / 2 \mathbb{Z} \longrightarrow \mathbb{Z}^{n} \rtimes \mathbb{Z} / 2 \mathbb{Z}$ l'homomorphisme défini par $s_{I}(1)=\left(i_{1}, \ldots, i_{n} ; 1\right)$. Il existe une unique classe

$$
\hat{v} \in H^{n}\left(\mathbb{Z}^{n} \rtimes \mathbb{Z} / 2 \mathbb{Z}, H_{n}\left(\mathbb{Z}^{n}\right)\right)
$$

relevant $v$ et telle que $s_{I}^{*}(\hat{v})=0$ pour tout $I$ contenant au moins un indice nul. De plus

$$
s_{(1, \ldots, 1)}(\hat{v})=1 \in H^{n}\left(\mathbb{Z} / 2 \mathbb{Z}, \mathbb{Z}^{n}\right)=\mathbb{Z} / 2 \mathbb{Z} .
$$

Preuve. Si $n=2 k$ ou $2 k+1$, la classe du $n$-cocycle

$c\left(\left(x_{11}, \ldots, x_{1 n} ; \alpha_{1}\right), \ldots,\left(x_{n 1}, \ldots, x_{n n} ; \alpha_{n}\right)\right)=(-1)^{\alpha} x_{11} \cdots x_{n n}, \quad \alpha=\alpha_{1}+\alpha_{3}+\cdots+\alpha_{2 k-1}$

satisfait les conditions du lemme. L'unicité de $\hat{v}$ résulte du lemme 3.2.2 en remarquant que toute section est conjuguée à une section du type $s_{I}$.

Preuve de la proposition 3.2.1. La classe $h(\ll x, y \gg)$ est représentée par la matrice $\operatorname{diag}\left(1, x, y, x^{-1} y^{-1}\right)=\xi \eta$, où $\xi=\operatorname{diag}\left(1, x, 1, x^{-1}\right)$ et $\eta=\operatorname{diag}\left(1,1, y, y^{-1}\right)$. L'homomorphisme $\varphi: \mathbb{Z}^{2} \longrightarrow S L(k)$ défini par $\varphi((1,0))=\xi$ et $\varphi((0,1))=\eta$ est $\mathbb{Z} / 2 \mathbb{Z}$-équivariant 
pour le changement de signe dans $\mathbb{Z}^{2}$ et l'involution $*$ dans $S L(k)$. On a donc un diagramme commutatif

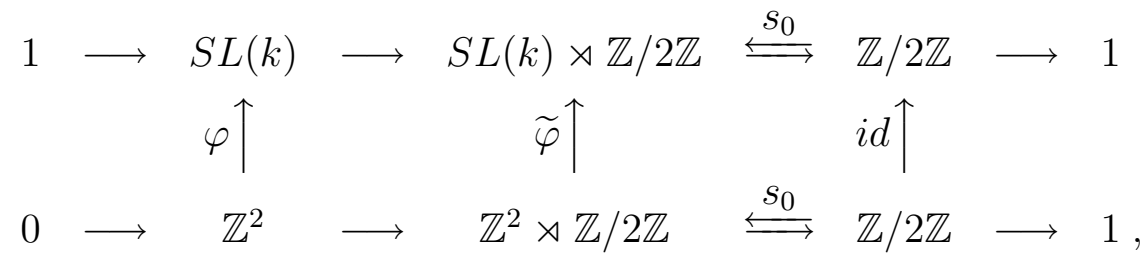

où $\widetilde{\varphi}=\varphi \rtimes i d$. Ce diagramme induit le diagramme commutatif

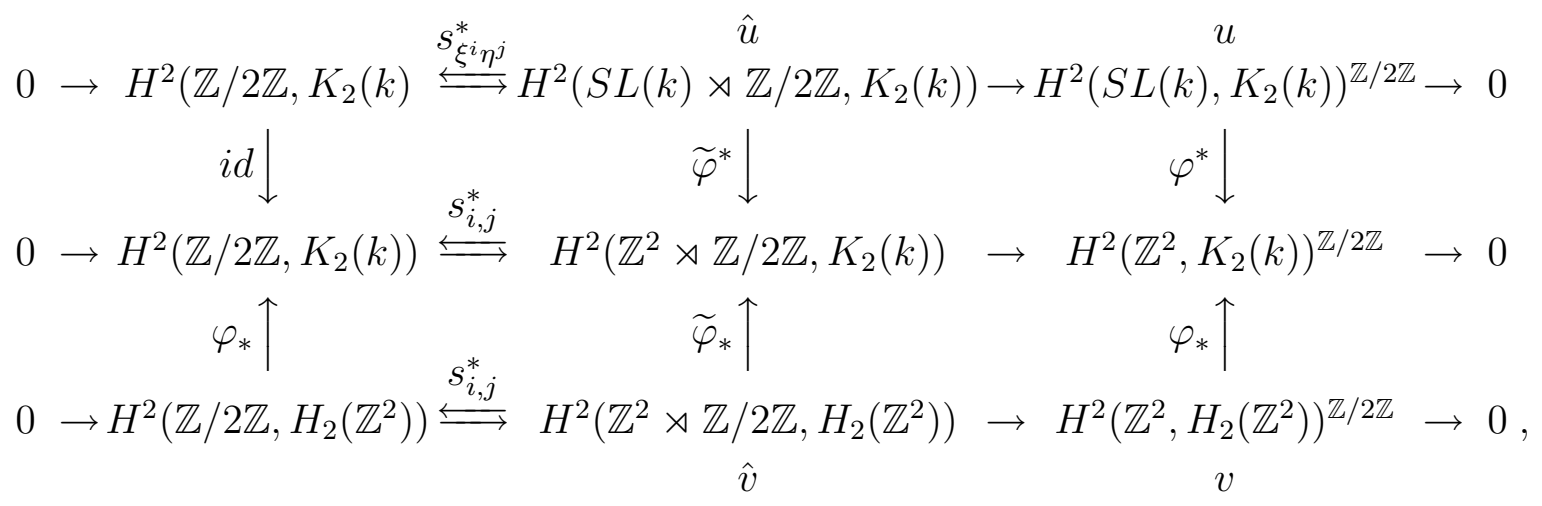

dans lequel la première ligne horizontale est exacte (mais, bien sûr, pas les autres).

Par définition le symbole

$$
\{x, y\} \in K_{2}(k) / 2 K_{2}(k)=H^{2}\left(\mathbb{Z} / 2 \mathbb{Z}, K_{2}(k)\right)
$$

est l'image de $1 \in H^{2}\left(\mathbb{Z} / 2 \mathbb{Z}, H_{2}\left(\mathbb{Z}^{2}\right)\right)=\mathbb{Z} / 2 \mathbb{Z}$ par $\varphi_{*}$. La proposition résulte donc de l'égalité

$$
\widetilde{\varphi}^{*}(\hat{v})=\widetilde{\varphi}_{*}(\hat{u}) \in H^{2}\left(\mathbb{Z}^{2} \rtimes \mathbb{Z} / 2 \mathbb{Z}, K_{2}(k)\right) .
$$

D'après le lemme 3.2.3 il suffit de démontrer que $s_{\xi}^{*}(\hat{u})=s_{2}(\xi)=0$ et $s_{\eta}^{*}(\hat{u})=s_{2}(\eta)=0$. Ceci est clair, car les formes $h^{-1}(\xi)$ et $h^{-1}(\eta)$ sont hyperboliques.

\subsection{Le troisième invariant.}

Soit $q$ un espace quadratique sur un anneau $A$, tel que $e_{1}(q)=0$ et $e_{2}(q)=0$. Puisque $s_{2}=\partial$ (proposition 2.2.1), $h(q)$ est dans l'image de $H^{1}(\mathbb{Z} / 2 \mathbb{Z}, S t(A)$ ) dans $H^{1}(\mathbb{Z} / 2 \mathbb{Z}, E(A))$. Le fait que $H_{1}(S t(A), \mathbb{Z})=H_{2}(S t(A), \mathbb{Z})=0$ ([MI], §5), permet de considérer l'invariant

$$
s_{3}: H^{1}(\mathbb{Z} / 2 \mathbb{Z}, S t(A)) \longrightarrow H^{3}\left(\mathbb{Z} / 2 \mathbb{Z}, H_{3}(S t(A))\right)
$$

Rappelons que $H_{3}(S t(A))$ n'est autre que $K_{3}(A)$ ([Lo], théorème 1.4.2). A tout choix d'une préimage de $h(q)$ dans $H^{1}(\mathbb{Z} / 2 \mathbb{Z}, S t(A))$ on peut donc associer un élément de $H^{3}\left(\mathbb{Z} / 2 \mathbb{Z}, K_{3}(A)\right)$. Nous ignorons si, pour un anneau quelconque, cet élément est indépendant du choix de la préimage de $h(q)$. Montrons que c'est bien le cas si $A$ est un corps $k$.

Proposition 3.3.1. Soit $k$ un corps. L'application $s_{3}$ se factorise par $H^{1}(\mathbb{Z} / 2 \mathbb{Z}, S L(k))$. 
Lemme 3.3.2. L'application

$$
S O(k)=H^{0}(\mathbb{Z} / 2 \mathbb{Z}, S L(k)) \stackrel{\partial}{\longrightarrow} H^{1}\left(\mathbb{Z} / 2 \mathbb{Z}, K_{2}(k)\right)={ }_{2} K_{2}(k)
$$

envoie tout $\alpha \in S O(k)$ sur le symbole $\{-1, N S(\alpha)\}$, où $N S(\alpha)$ est la norme spinorielle de $\alpha$.

Preuve. Pour simplifier les calculs il convient, dans cette démonstration, de remplacer les formes hyperboliques sur $k^{2 n}$ choisies au début par les formes hyperboliques

$$
\chi_{2 n}=\chi_{2} \perp \ldots \perp \chi_{2}, \quad \text { où } \quad \chi_{2}=\left(\begin{array}{ll}
0 & 1 \\
1 & 0
\end{array}\right) .
$$

L'involution * sur $S L(k)$ et sur $S t(k)$ sera donc à remplacer par l'involution $\alpha \mapsto \alpha^{\sharp}=$ $\chi_{2 n}\left(\alpha^{-1}\right)^{t} \chi_{2 n}$ sur $S L(k)$ et par son relèvement sur $S t(k)$. La conjugaison par une matrice $\omega \in G L_{2 n}(k)$ telle que $h_{2 n}=\omega^{t} \chi_{2 n} \omega$, induit un diagramme commutatif

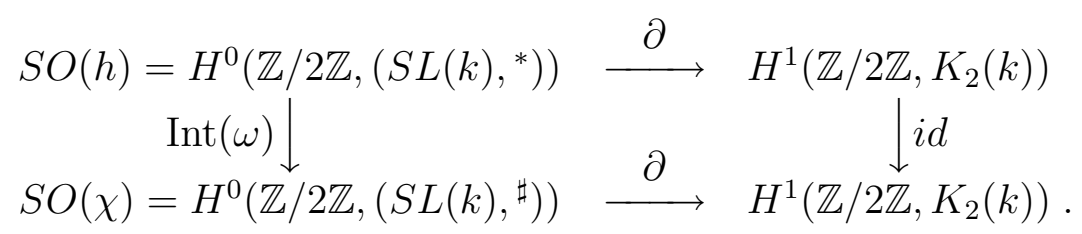

Puisque la norme spinorielle d'une matrice $\alpha$ dans $S O\left(h_{2 n}\right)$ est égale à celle de sa conjuguée $\omega \alpha \omega^{-1}$ dans $S O\left(\chi_{2 n}\right)$, il revient au même de démontrer la proposition pour l'une ou pour l'autre des involutions.

L'involution * sur $S t(k)$ satisfait, dans les notations de [9], les relations

et

$$
x_{12}(a)^{\sharp}=x_{12}(-a), \quad w_{12}(a)^{\sharp}=w_{12}(-a),
$$

On a donc

$$
h_{12}(a)^{\sharp}=w_{12}(a)^{\sharp} w_{21}\left(-a^{-1}\right)^{\sharp} w_{12}(-1)^{\sharp}=h_{12}(-a) w_{12}(1)^{2} .
$$

$$
h_{12}(a)^{-1} h_{12}(a)^{\sharp}=h_{12}(a)^{-1} h_{12}(-a) w_{12}(1)^{2}=\{-1, a\} h_{12}(-1) w_{12}(1)^{2}=\{-1, a\} .
$$

Comme $h_{12}(a)$ est une préimage dans $S t(k)$ de la matrice orthogonale

$$
\alpha=\left(\begin{array}{cc}
a & 0 \\
0 & a^{-1}
\end{array}\right)
$$

de norme spinorielle $a([\mathbf{1 1}], \S 55)$, ceci prouve que l'assertion est vraie pour les matrices de $\mathrm{SO}_{2}(k)$. Elle est vraie en général car, d'une part,

$$
S O_{2 n}=S O_{2}(k) \cdot\left[S O_{2 n}, S O_{2 n}\right]
$$

([6], Ch. VI, Theorem 6.3.3) et, d'autre part, aussi bien la norme spinorielle que $\partial$, étant des homomorphismes dans un groupe abélien, s'annulent sur le groupe des commutateurs de $S O_{2 n}$.

Preuve de la proposition. Deux classes $x, x^{\prime} \in H^{1}(\mathbb{Z} / 2 \mathbb{Z}, S t(k))$ ayant même image dans $H^{1}(\mathbb{Z} / 2 \mathbb{Z}, S L(k))$ peuvent être représentées par $\alpha, \alpha^{\prime} \in S t(k)$ satisfaisant $\alpha \alpha^{*}=\alpha^{\prime} \alpha^{\prime *}=$ 1 et $\alpha^{\prime}=c \cdot \alpha$ avec $c \in{ }_{2} K_{2}(k)$. Puisque la norme spinorielle est surjective et que, d'après [13], Theorem 1.8, tout élément de ${ }_{2} K_{2}(k)$ est de la forme $\{-1, u\}, c$ s'écrit $\beta^{-1} \beta^{*}$, où $\beta \in S t(k)$ se projette sur un élément orthogonal de $S L(k)$. La conjugaison par $\beta$ dans $S L(k)$ agit trivialement sur $K_{3}(k)$ et commute à l'involution * . On a donc

$$
s_{3}\left(\alpha^{\prime}\right)=s_{3}\left(\beta^{-1} \beta^{*} \alpha\right)=s_{3}\left(\beta^{*} \alpha \beta^{-1}\right)=s_{3}(\alpha) .
$$


Si $q$ est un espace quadratique sur un corps $k$, tel que $e_{1}(q)=e_{2}(q)=0$, la proposition 3.3.1 permet de définir $e_{3}(q)=s_{3}(\widetilde{h(q)})$, où $\widetilde{h(q)} \in H^{1}(\mathbb{Z} / 2 \mathbb{Z}, S t(k))$ est n'importe quelle préimage de $h(q) \in H^{1}(\mathbb{Z} / 2 \mathbb{Z}, S L(k))$. Nous allons maintenant comparer $e_{3}(q)$ avec l'invariant de Arason. Si $x, y, z \in k^{*}=K_{1}(k)$, nous notons $x \star y \star z \in K_{3}(k)$ le produit de ces éléments défini par Loday dans [7].

Théorème 3.3.3. Soit $q=\ll x, y, z \gg$ une forme de Pfister de rang 8 sur un corps $k$. Alors $e_{1}(q)=0, e_{2}(q)=0$ et $e_{3}(q)$ est égal à la classe dans $H^{3}\left(\mathbb{Z} / 2 \mathbb{Z}, K_{3}(k)\right)$ de l'élément $x \star y \star z$ de $K_{3}(k)$.

Preuve. Il est clair que $e_{1}(q)=0$. Pour démontrer les deux autres affirmations écrivons

$$
\begin{aligned}
q & =\ll x, y, z \gg=<1,-x>\otimes<1,-y>\otimes<1,-z> \\
& \simeq \operatorname{diag}\left(1,-x^{-1},-y^{-1}, x^{-1} y,-z^{-1}, x z^{-1}, y^{-1} z,-x y z\right)
\end{aligned}
$$

et, en accord avec les conventions faites au début de ce paragraphe,

$$
h(q)=\operatorname{diag}\left(1, x^{-1}, y^{-1}, x^{-1} y, z^{-1}, x z^{-1}, y^{-1} z, x y z\right)=\bar{\xi} \bar{\eta} \bar{\zeta},
$$

où

$$
\begin{aligned}
& \bar{\xi}=\operatorname{diag}\left(1, x^{-1}, 1, x^{-1}, 1, x, 1, x\right), \\
& \bar{\eta}=\operatorname{diag}\left(1,1, y^{-1}, y, 1,1, y^{-1}, y\right), \\
& \bar{\zeta}=\operatorname{diag}\left(1,1,1,1, z^{-1}, z^{-1}, z, z\right) .
\end{aligned}
$$

Il est clair que les invariants $s_{1}$ et $s_{2}$ sont nuls sur ces matrices (car elles sont des doubles). Elles se relèvent donc en trois éléments $\xi, \eta, \zeta \in S t(k)$, tels que $\xi^{*} \xi=\eta^{*} \eta=\zeta^{*} \zeta=1$. De plus, ces relevés commutent deux à deux pour la même raison et définissent donc un homomorphisme $\mathbb{Z} / 2 \mathbb{Z}$-équivariant $\varphi=\varphi_{\xi, \eta, \zeta}: \mathbb{Z}^{3} \longrightarrow S t(k)$. Cet homorphisme induit

$$
\varphi_{*}: H_{3}\left(\mathbb{Z}^{3}, \mathbb{Z}\right) \longrightarrow H_{3}(S t(k), \mathbb{Z})=K_{3}(k) .
$$

Nous montrons d'abord que $e_{3}(q)$ est égal à l'image par $\varphi_{*}$ d'un générateur du groupe cyclique $H_{3}\left(\mathbb{Z}^{3}, \mathbb{Z}\right)$. La méthode est complètement analogue à celle de la proposition 3.2.1. Il suffit d'utiliser le lemme 3.2.4 dans le cas $n=3$ et de démontrer le fait suivant.

Lemme 3.3.4.

$$
s_{3}(\xi \eta)=s_{3}(\eta \zeta)=s_{3}(\zeta \xi)=0 .
$$

Preuve. D'après 3.3.1 ces invariants ne dépendent que de $\bar{\xi} \bar{\eta}, \bar{\eta} \bar{\zeta}, \overline{\zeta \xi}$ dans $S L(k)$. Ils sont donc nuls, car les formes $h^{-1}(\bar{\xi} \bar{\eta}), h^{-1}(\bar{\eta} \bar{\zeta})$, et $h^{-1}(\overline{\zeta \xi})$ sont hyperboliques.

Il reste à démontrer que l'image par $\varphi_{*}$ d'un générateur $\omega$ de $H_{3}\left(\mathbb{Z}^{3}, \mathbb{Z}\right)$ est égale à $c=x \star y \star z$. D'après [7], exemple 2.2.8, $c$ est l'image de $\omega$ par $\Psi_{*}$, où $\Psi=\varphi_{\alpha, \beta, \gamma}$ et

$$
\begin{aligned}
& \bar{\alpha}=\operatorname{diag}\left(x, x^{-1}, 1,1, x^{-1}, x, 1,1,1,1, x, 1, x^{-1}\right), \\
& \bar{\beta}=\operatorname{diag}\left(y, 1, y^{-1}, 1, y, 1, y^{-1}, y^{-1}, y, 1,1,1,1\right), \\
& \bar{\gamma}=\operatorname{diag}\left(z, 1,1, z^{-1}, 1,1,1, z, 1, z^{-1}, z^{-1}, z, 1\right) .
\end{aligned}
$$

Appelons "disjoints" deux éléments de $E(A)$ ou de $S t(A)$ qui s'écrivent comme produits de générateurs $e_{i j}(a)$ ou $x_{i j}(a)$ sans indices communs. On constate qu'à une même permutation des lignes et des colonnes de $\bar{\xi}, \bar{\eta}, \bar{\zeta}$ près, on a $\bar{\alpha}=\overline{\xi \lambda}, \bar{\beta}=\overline{\eta \mu}, \bar{\gamma}=\bar{\zeta} \bar{\nu}$, où $\bar{\lambda}, \bar{\mu}, \bar{\nu}$, sont disjoints des autres et entre eux et se relèvent en des éléments $\lambda, \mu, \nu$ de $S t(k)$ disjoints entre eux et disjoints de $\xi, \eta$ et $\zeta$.

Soit, de façon générale, $\mathbb{N}=\mathrm{I} \cup \mathrm{J}$ une partition des entiers naturels en deux ensembles infinis disjoints et $A$ un anneau quelconque. Notons $S t_{\mathrm{I}}(A), S t_{\mathrm{J}}(A)$ les sous-groupes de $S t(A)$ engendrés par les $x_{i j}(a)$ avec $i, j \in \mathrm{I}$ et, respectivement, $i, j \in \mathrm{J}$. 
Lemme 3.3.4. Soient $\varphi: \mathbb{Z}^{3} \longrightarrow S t_{\mathrm{I}}(A)$ et $\psi: \mathbb{Z}^{3} \longrightarrow S t_{\mathrm{J}}(A)$ deux homomorphismes, définis par des triplets $\xi, \eta, \zeta$, respectivement $\lambda, \mu, \nu$, d'éléments qui commutent entre eux. Soit $\chi$ l'homomorphisme défini par le triplet $\xi \lambda, \eta \mu, \zeta \nu$. On a $\chi^{*} \omega=\varphi^{*} \omega+\psi^{*} \omega$.

Preuve. En choisissant des bijections $\mathbb{N} \longrightarrow \mathrm{I}$ et $\mathbb{N} \longrightarrow \mathrm{J}$ on obtient un homomorphisme

$$
\nabla: S t(A) \times S t(A) \longrightarrow S t(A)
$$

tel que $\chi$ est le composé

$$
\mathbb{Z}^{3} \stackrel{\delta}{\longrightarrow} \mathbb{Z}^{3} \times \mathbb{Z}^{3} \stackrel{\varphi \times \psi}{\longrightarrow} S t(A) \times S t(A) \stackrel{\nabla}{\longrightarrow} S t(A)
$$

Puisque

$$
H_{3}\left(\mathbb{Z}^{3} \times \mathbb{Z}^{3}\right)=\bigoplus_{i+j=3} H_{i}\left(\mathbb{Z}^{3}\right) \otimes H_{j}\left(\mathbb{Z}^{3}\right)
$$

$\chi^{*}$ se factorise par

$$
\bigoplus_{i+j=3} H_{i}(S t(A)) \otimes H_{j}(S t(A))
$$

et l'assertion suit du fait que $H_{1}(S t(A))=0$.

Ce lemme montre, d'une part que $c=\Psi^{*} \omega=\varphi^{*} \omega+\psi^{*} \omega$ et d'autre part que $\psi^{*} \omega=0$, car $\lambda, \mu$ et $\nu$ sont, à leur tour, disjoints deux à deux. On a donc démontré que $e_{3}(\ll x, y, z \gg)$ est la classe de $x \star y \star z$ dans $H^{3}\left(\mathbb{Z} / 2 \mathbb{Z}, K_{3}(k)\right)$.

\subsection{L'invariant de Arason.}

Pour faire le lien entre $e_{3}(q)$ et l'invariant $\operatorname{Ar}(q)$ de Arason dans le cas d'un espace quadratique $q$ défini sur un corps $k$ et tel que $e_{1}(q)=e_{2}(q)=0$, remarquons que l'isomorphisme $K_{3}^{M}(k) / 2 K_{3}^{M}(k) \longrightarrow H^{3}\left(k, \mu_{2}\right)$, établi par Rost [12] et par Merkur'ev et Suslin [8], fournit un homomorphisme de $H^{3}\left(k, \mu_{2}\right)$ dans $H^{3}\left(\mathbb{Z} / 2 \mathbb{Z}, K_{3}(k)\right)$ qui envoie $\operatorname{Ar}(q)$ dans $e_{3}(q)$.

\section{Bibliographie}

1. L. Evens: The Cohomology of Groups, Oxford, 1991.

2. C.H. Giffen: Hasse-Witt Invariants for $(\alpha, u)$-Reflexive Forms and Automorphisms. I: Algebraic $K_{2}$-Valued Hasse-Witt Invariants. J. Algebra 44 (1977), 434--456.

3. C.H. Giffen: Hermitian forms and algebraic K-Theory. Bull. A.M.S. 83 (1977), 13031305.

4. K.W. Gruenberg: The universal coefficient theorem in the cohomology of groups, J. London Math. Soc. 43 (1968), 239--341.

5. G. Hochschild and J-P. Serre: Cohomology of group extensions, Trans. Amer. Math. Soc. 74 (1953), 110-134.

6. M.-A. Knus: Quadratic and Hermitian Forms over Rings. Grundlehren der Math. Wissenschaften 294, Springer-Verlag, 1991.

7. J.-L. Loday: K-théorie algébrique et représentations de groupes. Ann. Sci. École Norm. Sup. 9 (1976), 309--377. 
8. A.S. Merkur'ev and A.A. Suslin: On the norm residue homomorphism of degree three. Izv. Akad. Nauk SSSR, Ser. Math. 54 (1990), 339--356 = Math. USSR Izvestiya 36 (1991), 349--367.

9. J. Milnor: Introduction to Algebraic K-Theory. Annals of Math. Studies 72, Princeton Univ. Press, 1971.

10. M. Ojanguren, R. Parimala and R. Sridharan: Ketu and the Second Invariant of a Quadratic Space. K-Theory, à paraître.

11. O.T. O'Meara: Introduction to Quadratic Forms. Grundlehren der Math. Wissenschaften 117, Springer-Verlag, 1963.

12. M. Rost: Hilbert 90 for $K_{3}$ for degree two extensions. Manuscrit, 1986.

13. A.A. Suslin: Torsion in $K_{2}$ of Fields. $K$-Theory 1 (1987), 5-29. 